\title{
Hemoglobina glicosilada y eventos cardiovasculares en pacientes diabéticos de un hospital universitario
}

\author{
Glycosylated hemoglobin and cardiovascular \\ events in diabetic patients of a university hospital
}

\author{
Claudia lucía Figueroa, Fabio Camilo Suárez, Andrés Felipe Ochoa, \\ LAURA JULIANA RENGIFO, JUAN RAMÓN ISAZA \\ - Bucaramanga, Santander (Colombia)
}

\section{Resumen}

Introducción: la prevalencia de comorbilidades cardiovasculares generadas por la diabetes mellitus, se desconoce a nivel global en Colombia y más a nivel local.

Objetivo: determinar la prevalencia de los eventos cardiovasculares según los niveles de hemoglobina glicosilada (HbA1c), en el Hospital Universitario de Santander.

Material y método: estudio observacional analítico retrospectivo tipo corte transversal con pacientes del servicio de medicina interna del Hospital Universitario de Santander en el año 2013.

Resultados: la prevalencia de diabetes fue $31.2 \%$ (HbA1c $\geq 6.5 \%$ ), de éstos, $52.2 \%$ fueron mujeres y la mayoría $(70.45 \%)$ recibían tratamiento farmacológico. Aunque el infarto agudo de miocardio (10.9\%) fue el evento más frecuente, la frecuencia de los diferentes eventos cardiovasculares como motivo de consulta no se relacionaron con algún nivel de HbA1c. Sin embargo, la HbA1c >9\% en pacientes $>65$ años tuvo tendencia de riesgo de ACV, pero sin significancia estadística. Entre los pacientes con $\mathrm{HbA1c}>9 \%, 62.02 \%$ recibían $\leq 4$ dosis/día de medicamentos $(p=0.000)$, con una prevalencia alta de muerte intrahospitalaria (88\%) siendo el OR de 2.08 (IC95\%: 0.85-5.1; $p=0.107)$. Se encontró en el análisis exploratorio, con algunas variables independientes relevantes cierto comportamiento predictivo de niveles de HbA1c no tan altos $\leq 9 \%$ (AROC 0.67) y otras, para muerte intrahospitalaria (AROC 0.7).

Conclusiones: en la población analizada la prevalencia de los eventos cardiovasculares en la población con $\mathrm{HbA} 1 \mathrm{c} \geq 6.5 \%$ fue $31.06 \%$, la categoría $\mathrm{HbA} 1 \mathrm{c}>9 \%$ se relacionó con mayor muerte intrahospitalaria; la $\mathrm{HbA} 1 \mathrm{c}<9 \%$ se relacionó con mayor dosis de tabletas para tratamiento. (Acta Med Colomb 2018; 43: 74-80).

Palabras clave: diabetes mellitus tipo 2; prevalencia; hemoglobina A glicosilada; infarto del miocardio; accidente cerebrovascular; enfermedad arterial periférica; insuficiencia cardiaca; mortalidad hospitalaria.

\section{Abstract}

Introduction: the prevalence of cardiovascular comorbidities generated by Diabetes Mellitus is unknown globally in Colombia and is further unknown locally.

Objective: to determine the prevalence of cardiovascular events according to the levels of glycosylated hemoglobin (HbA1c), at the University Hospital of Santander.

Material and Method: a cross-sectional, retrospective analytical observational study with patients from the internal medicine service of the University Hospital of Santander in 2013.

Results: the prevalence of diabetes was $31.2 \%$ ( $\mathrm{HbA} 1 \mathrm{c} \geq 6.5 \%$ ); of these $52.2 \%$ were women and the majority $(70.45 \%)$ received pharmacological treatment. Although acute myocardial infarction $(10.9 \%)$ was the most frequent event, the frequency of the different cardiovascular events as a reason for consultation was not related to any level of HbA1c. However, HbA1c>9\% in patients> 65 years had a risk of stroke, but without statistical significance. Among patients with $\mathrm{HbA} 1 \mathrm{c}>9 \%$, $62.02 \%$ received $\leq 4$ doses / day of medication $(\mathrm{p}=0.000)$, with a high prevalence of in-hospital

Dra. Claudia Lucía Figueroa: Especialista en Medicina Interna. MSc en Epidemiología Clínica. Docente Planta Posgrado Medicina Interna, Universidad Industrial de Santander, Hospital Universitario de Santander; Fabio Camilo Suárez-Cadena, Andrés Felipe Ochoa-Díaz y Laura Juliana Rengifo-Quintero: Estudiantes de Medicina, Miembros Grupo GERMINA-UIS; Dr. Juan Ramón Isaza-Angarita: Médico y Cirujano, Miembro Grupo GERMINA-UIS. Escuela de Medicina, Universidad Industrial de Santander, Bucaramanga. Santander (Colombia).

Correspondencia: Andrés Felipe Ochoa Díaz. Girón, Santander (Colombia).

E-mail: andresfelipe8adiaz@hotmail.com Recibido: 17 /I/ 2017 Aceptado: 4/IV/2018 
death $(88 \%)$ with an OR of 2.08 (95\% CI: $0.85-5.1 ; p=0.107)$. It was found in the exploratory analysis with some relevant independent variables certain predictive behavior of HbA1c levels not so high $\leq 9 \%$ (AROC 0.67) and others, for in-hospital death (AROC 0.7).

Conclusions: in the population analyzed, the prevalence of cardiovascular events in the population with $\mathrm{HbA} 1 \mathrm{c} \geq 6.5 \%$ was $31.06 \%$; the category $\mathrm{HbA} 1 \mathrm{c}>9 \%$ was associated with greater in-hospital death; HbA1c $<9 \%$ was associated with a higher dose of tablets for treatment. (Acta Med Colomb 2018; 43: 74-80).

Keywords: diabetes mellitus type 2; prevalence; glycosylated hemoglobin; myocardial infarction; cerebrovascular accident; peripheral arterial disease; heart failure; hospital mortality.

\section{Introducción}

En Colombia se desconoce la prevalencia exacta de diabetes mellitus 2 (DM2), debido a la escasez de estudios poblacionales, por consiguiente la frecuencia de la patología cardiovascular en estos pacientes también se ignora. Entre algunos reportes de esta situación en la población diabética nacional se encuentra el estudio CARMELA (1), que midió la prevalencia de diversos factores de riesgo cardiovascular en seis ciudades de Latinoamérica, hallándose una prevalencia de DM2 en personas de 25-64 años en Bogotá de 7.4\% en hombres y $8.7 \%$ en mujeres. Sin embargo, el primer estudio que se realizó acerca de este tema fue publicado en 1993 y encontró una prevalencia de $7.3 \%$ en hombres y $7.4 \%$ en mujeres mayores de 30 años (2). Es importante mencionar que la prevalencia de DM2 aumenta desde $3 \%$ en personas de 30-39 años, hasta 20\% en adultos de 70-80 años. Por lo tanto, su frecuencia aumenta en poblaciones con mayor proporción de personas adultas.

Hasta el momento se considera que dentro de las comorbilidades generadas por la diabetes, la enfermedad cardiovascular es la que más afecta la calidad de vida y disminuye la sobrevida de este tipo de pacientes, causando eventos patológicos tempranos y alterando capacidades físicas a futuro. La evidencia de las lesiones se aprecia desde que se hace el diagnóstico de DM2, debido a que en ese momento 16-21\% de los pacientes ya presentan retinopatía, 12-23\% nefropatía y $25-40 \%$ neuropatía $(3,4)$. Posteriormente aparecen las complicaciones macrovasculares (síndrome coronario agudo, enfermedad cerebrovascular isquémica, enfermedad arterial periférica), como consecuencia de trastornos endoteliales, ayudado por otros factores de riesgo metabólicos y conductuales del individuo, como hipertensión arterial (HTA), dislipidemia, sedentarismo y tabaquismo.

Es importante encontrar o identificar rangos entre las pruebas de laboratorios que apunten u orienten en la detección de enfermedades de alta prevalencia con comorbilidades a largo plazo, ya que los altos gastos de atención que demandan los pacientes diabéticos llegan a ser el $11 \%$ del presupuesto total de salud en Estados Unidos y, alrededor de $6 \%$ del costo de salud en países de América Latina y el Caribe (5-7).

En consecuencia, es importante mencionar que la presencia de eventos cardiovasculares asociados a DM2, reflejan de forma indirecta la oportunidad en el diagnóstico precoz, en el manejo oportuno y en la adherencia del paciente al tratamiento. De manera que, medir la prevalencia de eventos cardiovasculares en la población diabética que asiste al Hospital Universitario de Santander (HUS) constituye un indicador de cumplimiento de metas de tratamiento, lo cual puede servir en futuras investigaciones que busquen hacer inferencias sobre los puntos a fortalecer en el manejo del paciente diabético. Es por lo anterior que el presente estudio tiene como objetivo-determinar la prevalencia de los eventos cardiovasculares según los niveles de hemoglobina glicosilada (HbA1c), en el Hospital Universitario de Santander.

\section{Población de estudio}

\section{Material y métodos}

Se realizó un estudio observacional analítico retrospectivo tipo corte transversal en pacientes que ingresaron al servicio de urgencias de medicina interna del HUS en el año 2013. El tamaño de muestra se obtuvo por conveniencia a través de un muestreo no probabilístico con el fin de obtener todo el universo probable entre las estadísticas de los últimos dos años (2011 y 2012) de pacientes con HbA1c $\geq 6.5 \%$ ( $n=250$ por año). Se incluyeron a todos los pacientes que hubiesen consultado desde el 01 de enero hasta 31 de diciembre de 2013 y que tuvieran una medición de HbA1c mayor o igual a $6.5 \%$ (definición guías American Diabetes Association). Como criterios de exclusión se consideraron a pacientes en estado de embarazo y a menores de 18 años de edad.

El HUS es un hospital de tercer nivel de complejidad de carácter público, que presta servicios de mediana y alta complejidad a la población del nororiente colombiano, principalmente al departamento de Santander y a otros como Bolívar, Cesar, Norte de Santander y Arauca. Esta institución se encuentra catalogada como sitio de prácticas de carácter universitario, y cuenta con convenio docencia-servicio con la Universidad Industrial de Santander.

Se obtuvo una base de datos a través del servicio de estadística del hospital que contenía los resultados de las mediciones de HbA1c en el año de estudio, posteriormente se seleccionaron los pacientes con resultados que cumplieron con los criterios de inclusión y se procedió a consultar la historia clínica de cada paciente para extraer los datos necesarios. 


\section{Variables}

Se establecieron variables sociodemográficas como sexo, edad, estado civil, escolaridad, ocupación y lugar de residencia según el registro en la historia clínica electrónica. Por otra parte, se registraron los datos correspondientes a los pacientes, que en el servicio se les definió diagnóstico de patologías según criterio médico y/o criterio de laboratorio y/o criterio imagenológico que figuraran en la historia clínica electrónica, entre éstas están: infarto agudo del miocardio (IAM), enfermedad arterial periférica (EAP), insuficiencia cardiaca congestiva (ICC) y accidente cerebrovascular (ACV). Además, se tomaron aquellos datos de la historia clínica, que el médico tratante registraba como antecedente de IAM, EAP, ICC, ACV, HTA, dislipidemias, tabaquismo y tiempo de diagnóstico de DM2. También, se registraron variables relacionadas a la terapia farmacológica como la existencia de tratamiento para la DM2, o para otras enfermedades, la frecuencia del uso de medicamentos por día medidos en número de formas farmacéuticas (tabletas o dosis de insulina) (dosis/día), teniendo en cuenta diversas formas farmacéuticas y vías de administración). Junto a lo anterior, se tuvo en cuenta el tiempo de estancia hospitalaria en meses, tiempo del diagnóstico de la DM2, los niveles en HbA1c y el desenlace de muerte intrahospitalaria.

\section{Análisis estadístico}

Se realizó en primer lugar un análisis univariado teniendo en cuenta el tipo de variable continua o categórica, definiendo sus medidas de frecuencia (porcentajes, medias, medianas) y unidades de dispersión (desviación estándar, intervalos de confianza, rangos intercuartílicos) según correspondiera. En segundo lugar, se exploró un análisis bivariado donde se midieron diferencias y significancias estadísticas según el tipo de variable, ttest, chi $^{2}$, análisis de asociación con regresión logística e identificando ajustes del modelo. Por último, se indagó un modelo multivariado con las variables que estadísticamente lo permitieron, con OR e intervalos de confianza del $95 \%$ significativos, definiendo la significancia estadística como $p<0,05$, obteniéndose además un modelo predictor con área bajo la curva (AROC).

\section{Consideraciones éticas}

El estudio fue realizado con el aval de los comités de ética de la Universidad Industrial de Santander y el HUS. El desarrollo del estudio se basó en la normatividad expuesta en la Declaración de Helsinki para principios éticos en las investigaciones médicas en seres humanos, adicionalmente se tomaron en cuenta la Resolución 8430 de 1993 del Ministerio de Salud y la Ley Estatutaria 1581 de 2012 de Colombia, según las cuales esta investigación corresponde a un estudio sin riesgos, manteniendo siempre la protección de los datos personales, derechos y privacidad de los pacientes.

\section{Resultados}

\section{Análisis descriptivo o univariado}

En el HUS fueron solicitados un total de 898 exámenes cuantitativos de HbA1c de enero a diciembre de 2013, se presentó una prevalencia de $31.2 \%(\mathrm{n}=281)$ de $\mathrm{DM}(\mathrm{HbA} 1 \mathrm{c}$ $\geq 6.5 \%$ ). El promedio de $\mathrm{HbA} 1 \mathrm{c}$ de los pacientes estudiados fue de $9.60 \%$ (DE 2.29\%). El promedio de edad fue 60.4 \pm 15 años, el $52.27 \%(n=138)$ eran mujeres, y la mayoría residía dentro del casco urbano. El 70.45\%(n=186) recibían tratamiento para la DM y el $23.85 \%(n=63)$ no lo recibía. Dentro de los motivos de consulta de origen cardiovascular, el más prevalente fue el IAM 10.98\% (n=29). El 68.93\% ( $\mathrm{n}=182)$ correspondió a un motivo de consulta de origen no cardiovascular. De la misma manera, el IAM representó el antecedente de evento cardiovascular más prevalente dentro de la serie (Tabla 1).

Por otra parte, $50.89 \%(n=57)$ de aquellos pacientes en quienes se documentó el tiempo desde el diagnóstico de DM, este último estuvo entre 1 y 5 años. La mediana del tiempo de la DM fue cinco años, con un valor mínimo de un mes y un máximo de 30 años. Adicionalmente, $57.6 \%(n=152)$ consumían de 0-3 dosis de medicamentos al día, con una

Tabla 1. Caracterización de la población.

\begin{tabular}{|c|c|c|c|}
\hline Variable & Categorías & Número & Porcentaje (\%) \\
\hline \multirow{2}{*}{ Sexo } & Masculino & 126 & 47.73 \\
\hline & Femenino & 138 & 52.27 \\
\hline \multirow{5}{*}{ Escolaridad } & Ninguna & 6 & 2.27 \\
\hline & Primaria & 26 & 9.85 \\
\hline & Secundaria & 6 & 2.27 \\
\hline & Profesional & 0 & 0 \\
\hline & No hay datos & 226 & 85.61 \\
\hline \multirow{5}{*}{ Ocupación } & Empleado & 4 & 1.52 \\
\hline & Desempleado & 15 & 5.68 \\
\hline & Hogar & 23 & 8.71 \\
\hline & Independiente & 19 & 7.20 \\
\hline & No hay datos & 203 & 76.89 \\
\hline \multirow{4}{*}{ Antecedentes* } & IAM & 42 & 15.91 \\
\hline & $\mathrm{ACV}$ & 21 & 7.95 \\
\hline & EAP & 38 & 14.39 \\
\hline & ICC & 34 & 12.88 \\
\hline \multirow{3}{*}{$\begin{array}{l}\text { Factores de } \\
\text { riesgo } \mathrm{CV}\end{array}$} & HTA & 138 & 52.27 \\
\hline & Dislipidemia & 52 & 19.70 \\
\hline & Tabaquismo & 72 & 27.27 \\
\hline \multirow{6}{*}{$\begin{array}{l}\text { Motivos de } \\
\text { consulta* }\end{array}$} & IAM & 29 & 10.98 \\
\hline & $\mathrm{ACV}$ & 14 & 5.30 \\
\hline & EAP & 23 & 8.71 \\
\hline & ICC & 1 & 4.55 \\
\hline & HTA & 4 & 1.52 \\
\hline & No CV & 182 & 68.94 \\
\hline \multirow{7}{*}{$\begin{array}{l}\text { Numero de } \\
\text { dosis/día }\end{array}$} & 0 & 76 & 28.79 \\
\hline & $1-3$ & 76 & 28.79 \\
\hline & $4-6$ & 61 & 23.11 \\
\hline & $7-9$ & 31 & 11.74 \\
\hline & $10-12$ & 12 & 4.55 \\
\hline & $13-15$ & 7 & 2.65 \\
\hline & 16 & 1 & 0.38 \\
\hline \multicolumn{4}{|c|}{$\begin{array}{l}\text { Me: Mediana. CV: cardiovascular. IAM: infarto agudo del miocardio. ACV: accidente } \\
\text { cerebrovascular. EAP: enfermedad arterial periférica. ICC: insuficiencia cardiaca } \\
\text { congestiva. HTA: hipertensión arterial. } \\
\text { *Los valores corresponden a los pacientes con el antecedente o motivo de consulta } \\
\text { positivo. }\end{array}$} \\
\hline
\end{tabular}


mediana de cuatro dosis por día (RIQ 2-6 dosis/día) y con un valor mínimo de 0 dosis/día y un máximo de 15 dosis/ día. El 50.4\% $(\mathrm{n}=177)$ presentaron una estancia hospitalaria entre 1 y 10 días, y para el total de la muestra la mortalidad fue de $8.71 \%(n=23)$ (Tabla 1).

La prevalencia de eventos cardiovasculares en el grupo estudiado, según rangos de HbA1c definidos cada $0.5 \%$, a partir de $6.5 \%$ se detalla en la Tabla 2.

\section{Análisis bivariado}

Con respecto a los motivos de consulta de tipo cardiovascular, se destacó que los pacientes diabéticos mayores de 65 años tuvieron seis veces más probabilidad de consultar por ACV (OR 6.29; IC=1.71-23; $p=0.006$ ). Al explorar la probabilidad de encontrar relación entre niveles de $\mathrm{HbA} 1 \mathrm{c}$ y antecedentes cardiovasculares se relacionó $\mathrm{HbAlc} \leq \mathrm{de}$ $8 \%$ en los pacientes con antecedentes de ICC e HTA, con $p=0.042$ y $p=0.001$, respectivamente.

Las personas con niveles altos de HbA1c (>9\%), en promedio consumían 3 dosis/día de medicamentos; aquellas con niveles intermedios (HbA1c 8-9\%) 4 dosis/día de medicamentos y los que tenían niveles menores $(\leq 8 \%)$ consumían en promedio 6 dosis/día de medicamentos $(p=0.000)$. Respecto a la relación entre $\mathrm{HbA} 1 \mathrm{c}$ categorizada en los tres grupos ya mencionados y la edad; en mayores de 65 años el $46.6 \%$ tenían niveles de $\mathrm{HbA} 1 \mathrm{c} \leq 8 \%$; mientras que en los pacientes menores o iguales a 65 años el $40.9 \%$ tenían niveles $>9 \%(p=0.004)$.

Entre la población analizada, se evidenció que el $88 \%$ $(\mathrm{n}=114)$ de los fallecidos se relacionaba con niveles de HbA1c $>9 \%(p=0.10)$, relación estadísticamente débil, que tampoco se relacionó con los demás motivos de consulta analizados. Posteriormente, al estimar la relación entre el consumo de medicamentos ( $\leq 4$ dosis/día y $>4$ dosis/día) y la $\mathrm{HbA} 1 \mathrm{c}$, el $62,02 \%$ de los pacientes con HbA1c mayor de $9 \%$ recibían menos o igual a 3 dosis/día $(p=0,000)$.

\section{Análisis exploratorio multivariado}

Después del análisis crudo bivariado, se estimaron posibles tendencias de asociación entre las variables independientes que dieron estimaciones con significancia estadística como el consumo de medicamentos, y las variables de desenlace HbAlc $(\leq 9 \%$ y $>9 \%)$ y desenlace de muerte.

$\mathrm{Al}$ analizar el consumo de medicamentos ( $>4$ dosis/día; $\leq 4$ dosis/día) con la HbAlc dicotomizada $(\leq 9 ;>9 \%)$ se encontró que más de 4 dosis/día se asociaba un $64 \%$ más con $\mathrm{HbA} 1 \mathrm{c}<9 \%$ (OR=0.36 IC95\%:0.21-0.59; $p=0.000$ ). Adicionalmente, no se encontró asociación entre la variable consumo de medicamentos con antecedentes o motivos de consulta tipo cardiovascular.

Se exploraron también dos modelos de predicción, el primero de predicción de bajos niveles de $\mathrm{HbA} 1 \mathrm{c}(\mathrm{HbA} 1 \mathrm{c} \leq 9 \%)$ con las variables edad $>65$ años y consumo de $>4$ dosis/día de medicamentos con OR en valores de protección, obteniéndose una AROC de 0.67. Y el segundo, de predicción
Tabla 2. Número de eventos cardiovasculares registrados como motivos de consulta según niveles de $\mathrm{HbAlc}$.

\begin{tabular}{|l|c|c|c|c|c|}
\hline Niveles HbA1c (\%) & IAM & HTA & ICC & ECV & EAP \\
\hline$\geq 6,5 \%-7 \%$ & 0 & 0 & 0 & 0 & 0 \\
\hline$\geq 7 \%-<7,5 \%$ & 6 & 1 & 4 & 6 & 3 \\
\hline$\geq 7,5 \%-<8 \%$ & 0 & 0 & 1 & 0 & 0 \\
\hline$\geq 8 \%-<8,5 \%$ & 3 & 1 & 2 & 1 & 6 \\
\hline$\geq 8,5 \%-<9 \%$ & 2 & 0 & 0 & 1 & 0 \\
\hline$\geq 9 \%$ & 17 & 2 & 5 & 6 & 15 \\
\hline
\end{tabular}

Tabla 3. Análisis multivariado, modelo de predicción de bajos niveles de HbAlc.

\begin{tabular}{|l|c|c|c|c|}
\hline HbA1C $\leq 9 \%$ & OR & $p$ & \multicolumn{2}{|c|}{$\begin{array}{c}\text { 95\% intervalo de } \\
\text { confianza }\end{array}$} \\
\hline Edad $>65$ años & 0.50 & 0.011 & 0.30 & 0.85 \\
\hline $\begin{array}{l}\text { Consumo de } \\
\text { medicamentos } \\
>4 \text { dosis/día }\end{array}$ & 0.39 & 0.000 & 0.23 & 0.65 \\
\hline
\end{tabular}

Tabla 4. Análisis multivariado, modelo de predicción de muerte intrahospitalaria.

\begin{tabular}{|l|c|c|c|c|}
\hline \multicolumn{1}{|c|}{$\begin{array}{c}\text { Desenlace de } \\
\text { muerte }\end{array}$} & OR & $\boldsymbol{p}$ & \multicolumn{2}{c|}{$\begin{array}{c}\text { 95\% intervalo de } \\
\text { confianza }\end{array}$} \\
\hline $\mathrm{HbA} 1 \mathrm{c}>9 \%$ & 2.46 & 0.05 & 0.96 & 6.28 \\
\hline Ant. ACV & 3.79 & 0.025 & 1.18 & 12.17 \\
\hline Ant. Tabaquismo & 1.97 & 0.016 & 1.13 & 3.43 \\
\hline Ant: Antecedente.
\end{tabular}

de muerte intrahospitalaria, con las variables la $\mathrm{HbA} 1 \mathrm{c}>9 \%$, antecedente de ACV y de tabaquismo, con una AROC de 0.70 (Tablas 3, 4), (Figuras 1, 2).

\section{Discusión}

Caracterizar la población diabética asistente a una institución de tercer nivel, de mayoría régimen subsidiado, podría sugerir poblaciones muy susceptibles, débiles o de peores pronósticos; sin embargo, como en la población general, algunos lo están haciendo bien y otros mal. Aunque la muestra analizada no permitió identificar tendencia de eventos cardiovasculares, sí permitió caracterizar sus antecedentes, estado metabólico por $\mathrm{HbA} 1 \mathrm{c}$, dosis de tratamientos y evolución. Un estudio realizado en un hospital de Bogotá, Colombia, en pacientes diabéticos, evidenció una prevalencia de hospitalizaciones por eventos cardiovasculares de $13 \%$, siendo el principal motivo de consulta la HTA; además sugirió una relación entre enfermedad coronaria y pobre control metabólico (definido como HbA1c >7\%) (8).

Respecto a los pacientes con ICC, según lo reportado en dos estudios la media de $\mathrm{HbA} 1 \mathrm{c}$ en quienes tenían niveles de esta última $<8 \%$ osciló entre 7.4 y $7.6 \%(9,10)$, lo cual 


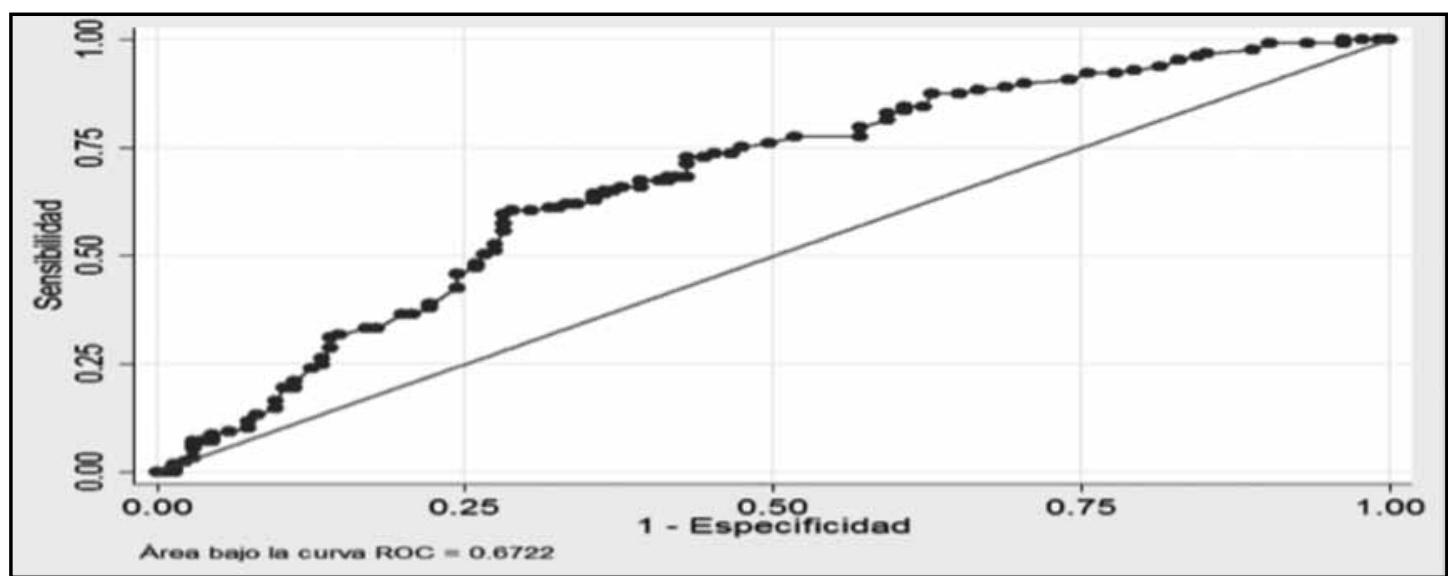

Figura 1. Curva ROC modelo de predicción de bajos niveles de HbAlc.

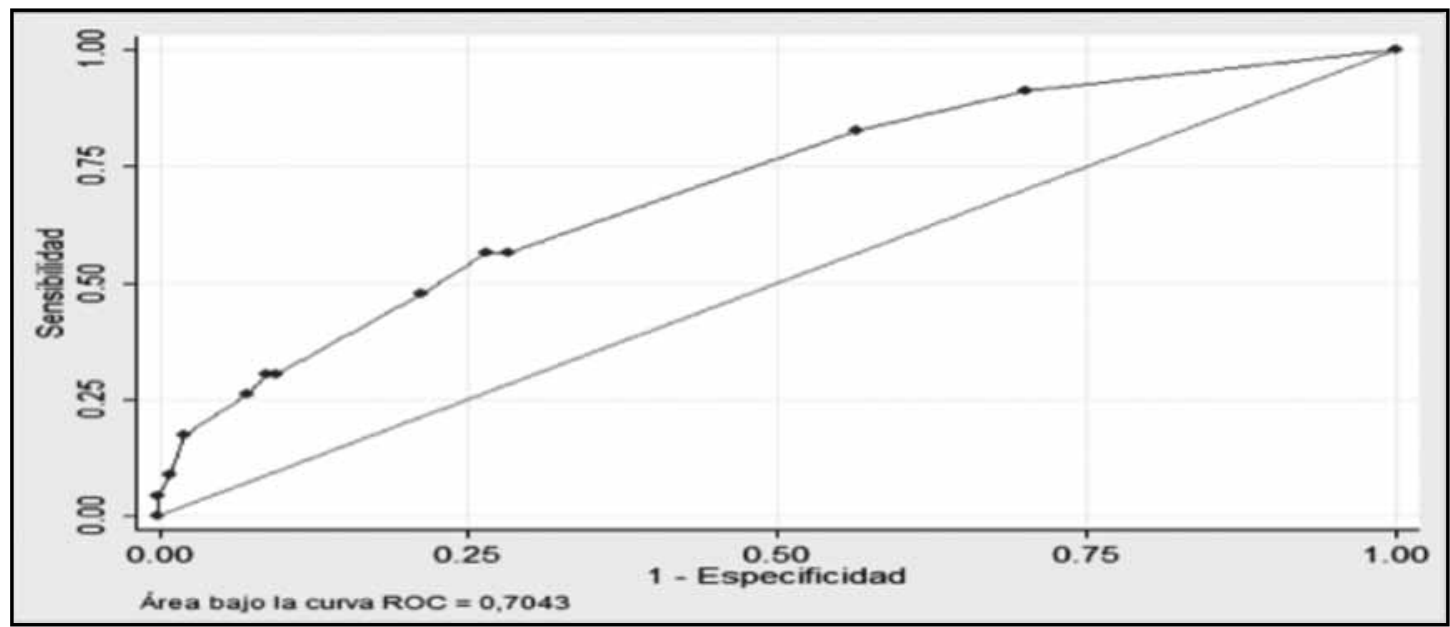

Figura 2. Curva ROC modelo de predicción de muerte intrahospitalaria.

coincide con el presente estudio donde se evidenció que más de la mitad tenían niveles $<8 \%$. No obstante, Skrtic S et al (9) encontraron un comportamiento diferente respecto a los niveles $<8 \%$ en una población similar, donde solo $21 \%$ de los pacientes con ICC tuvo estos niveles de HbA1c versus $55-88 \%$ del actual estudio. Además, este autor también reportó $23 \%$ de pacientes con niveles de $\mathrm{HbA} 1 \mathrm{c}$ altos $(>10 \%)$, lo cual es similar a los resultados de la población del HUS (20\%). En el ámbito nacional, Osuna $\mathrm{M}$ et al encontraron un modelo de asociación de riesgo de mortalidad teniendo en cuenta una edad $>75$ años, la concentración de HbA1c $>7 \%$ y la presencia de ICC (8). Y por otro lado en Bucaramanga, Colombia, se realizó un estudio en pacientes con DM2 y un IAM previo, que no evidenció una relación significativa entre la HbA1c y la fracción de eyección del ventrículo izquierdo, sin embargo, encontró que la HbA1c >8.5\% tuvo tendencia a relacionarse con fracciones de eyección del ventrículo izquierdo $<50 \%$ ( $p=0.019)$ (11).

Adicionalmente en el presente estudio se encontró que $39.86 \%$ de los pacientes con antecedente HTA presentaron niveles de $\mathrm{HbA} 1 \mathrm{c}<8 \%$, mientras que $23.91 \%$ tuvieron niveles altos siendo la diferencia significativa $(\mathrm{p}=0.001)$. Según Margolis KL et al(12), los pacientes con DM2 en control estricto o estándar de HTA y con control estándar de la glicemia presentaron valores de HbA1c de 7.5\%. Así mismo, Zoungas S et al (13), reportaron valores similares en su cohorte de pacientes, donde evidenció que los niveles de HbA1c en los pacientes con DM2 e HTA en seguimiento estándar reportaban valores de HbA1c del 7.5\%, similar a los encontrados en este estudio. Por otro lado, se ha evidenciado en pacientes con pobre control glicémico (definido por Bower JK et al en su estudio como HbA1c $\geq 7 \%$ ) (14) un riesgo de 1.25 veces (IC95\%:1.01-1.56) de HTA incidente.

Respecto al consumo de más de cuatro tabletas de medicamentos al día se puede inferir que los pacientes tienen mejor apoyo o entendimiento respecto al tratamiento, relacionándose esto con mayor adherencia lo que les permite tener menores niveles de HbA1c. Según esto, un estudio realizado en Holanda evidenció una diferencia significativa $(p<0.0001)$ entre los pacientes adherentes al tratamiento de 
DM2 y los no adherentes durante un tratamiento de un año con medias de $\mathrm{HbA} 1 \mathrm{c}$ de $6.9 \%$ y $7.2 \%$, respectivamente(15). Adicionalmente, existen publicaciones que demuestran las variables más relacionadas con el no control metabólico; un estudio de los factores asociados a la falta de control metabólico en pacientes con DM2 realizado en Bucaramanga, Colombia, mostró que la falta de adherencia al tratamiento tiene un RP=1.6 (IC95\%: 1.01-1.34; $p<0.05$ ) para no tener control metabólico(16). Por otro lado, una revisión sistemática que incluyó 36 trabajos evidenció que consumir más de un medicamento para la DM2 o más de dos dosis al día de fármacos son barreras para que un paciente alcance el control metabólico (17).

Hasta el día de hoy se han realizado tres grandes ensayos clínicos del control de la glicemia y su relación con eventos cardiovasculares (Advance Accord, VADT). En ellos se encontró una reducción significativa del riesgo cardiovascular de $10-17 \%(18,19)$ y de la HbA1c a $6.5 \%(19)$, en los pacientes que tenían un estricto seguimiento glucémico y especialmente en los de edad avanzada permitiendo relacionar con los hallazgos de este estudio en el que se presentaron mejores niveles de $\mathrm{HbA} 1 \mathrm{c}$ en mayores de 65 años. Esto probablemente sea resultado de una adherencia adecuada al tratamiento farmacológico evidenciada por el consumo de más de cuatro tabletas de medicamentos al día, a mejores hábitos de vida o a un tratamiento adecuado desde el diagnóstico. No se ha demostrado o mencionado que la edad sea un factor que modifique los valores de HbA1c, ya que estos se ven alterados en casos puntuales como valores extremos de hemoglobina total o situaciones en las que el recambio eritrocitario se encuentra aumentado (20). Por tanto, la edad no constituye una explicación por sí misma para la evidencia de estos hallazgos.

También se encontró en la presente investigación un aumento de la probabilidad de riesgo para mortalidad intrahospitalaria con los niveles de $\mathrm{HbAlc}$ aunque sin significancia estadística (OR de 2.08, $p=0.107$ ), esto es similar a lo descrito en múltiples estudios en los que evaluaron esta asociación con eventos cardiovasculares. Masrur et al (21) incluyeron 58265 pacientes con ACV hallando que niveles de $\mathrm{HbA} 1 \mathrm{c}$ mayores de $6.5 \%$ aumentan la mortalidad intrahospitalaria $\mathrm{OR}=1.36$ (IC95\%:1.211.53) $\mathrm{p}<0.0001$. También la mortalidad intrahospitalaria es mayor en pacientes con IAM y niveles de HbAlc altos $\mathrm{OR}=1.5$ (IC95\%:1.15-1.9; $p=0.002$ ) (22), y aquellos que no conocían su estado de diabetes tuvieron una mortalidad de $16.7 \%$ contra los que sí sabían y estaban en tratamiento (8.4\%) (23). Es tal el grado de asociación de la HbA1c con la mortalidad que algunos estudios basados en pacientes en Unidad de Cuidados Intensivos plantean la necesidad de evaluar los niveles al ingreso debido a que aquellos con $\mathrm{HbA} 1 \mathrm{c} \geq 6,5 \%$ y que no conocen su estado de diabetes han mostrado mayor mortalidad OR=3.37 (IC95\%: 1.60-7.11, $p=0.001)(24,25)$. Esta relación también se encuentra en patologías como la pancreatitis aguda donde los pacientes con $\mathrm{HbA} 1 \mathrm{c}$ alta tienen una mortalidad de $16 \%$ que difiere significativamente respecto a los no diabéticos (24). Sin embargo, algunas publicaciones no concuerdan respecto a los hallazgos del actual estudio, por lo que la asociación entre mortalidad intrahospitalaria y $\mathrm{HbA} 1 \mathrm{c}$ no es totalmente consistente ya que en pacientes con IAM en algunos casos no encuentran diferencias en mortalidad(26-28); por ejemplo Tian et al(27) clasificaron a los pacientes en tres grupos respecto a niveles de $\mathrm{HbAlc}$, no encontrando a los siete días diferencias significativas en mortalidad $(p=0.179)$.

$\mathrm{Al}$ ser un estudio retrospectivo no se es ajeno a los sesgos de información y clasificación, sin embargo, la extracción objetiva y el análisis exhaustivo de la historia clínica junto a los paraclínicos, permitieron una mejor validez estadística de los objetivos. Además, es importante resaltar el sesgo de selección que puede afectar datos como la prevalencia de DM al excluir pacientes diabéticos controlados en tratamiento con niveles de $\mathrm{HbA} 1 \mathrm{c}$ inferiores a $6.5 \%$; y el sesgo de clasificación al utilizar una sola herramienta de medición de niveles de glicemia. Se observa que se tiene un comportamiento muy extrapolable pero que amerita complementariedad y unificación en los registros clínicos, así como retroalimentación constante con los sistemas o programas de atención de este factor de riesgo cardiovascular, para que identifiquen y tomen medidas más tempranas sobre los factores predictores de severidad en diabetes.

Fue posible observar que a mayor concentración de HbAlc, hubo mayor probabilidad de eventos cardiovasculares, tal cual se observó en el análisis exploratorio bivariado y multivariado, siendo una estimación que amerita estudios en el futuro con mayor control de sesgos. Además, podría plantearse la necesidad de realizar una investigación con objetivos definidos que identifiquen verazmente medidas de efecto, con muestras y diseños que establezcan más que tendencias, asociaciones entre los niveles de $\mathrm{HbAlc}$ con eventos cardiovasculares debido a que la HbA1c es un marcador de diabetes y daño endovascular. Según los resultados el consumo de medicamentos es un factor que predice menores niveles de $\mathrm{HbA} 1 \mathrm{c}$ por lo que se puede investigar la asociación de eventos cardiovasculares con el uso de medicamentos específicos. Finalmente se propone para futuras investigaciones validar la capacidad discriminativa de los modelos de asociación hallados en este estudio.

\section{Conclusiones}

En el HUS se encontró que la prevalencia de DM2 es $31.2 \%$ para el año 2013 , y la prevalencia de eventos cardiovasculares fue de $31.06 \%$. El antecedente de ICC y HTA se asociaron con niveles de HbA1c menores de 9\%, sin encontrarse relación de la HbAlc con los demás eventos cardiovasculares evaluados como antecedentes o motivos de consulta. Por otro lado, el estudio permitió generar dos modelos de predicción uno de riesgo de muerte intrahospitalaria con niveles altos de $\mathrm{HbA} 1 \mathrm{c}$, y otro de protección para niveles bajos de $\mathrm{HbA1c}$. 


\section{Financiación}

La investigación aquí evidenciada no requirió financiación por parte de los autores ni por agentes externos.

\section{Referencias}

1. Escobedo J, Buitrón LV, Velasco MF, Ramírez JC, Hernández R, Macchia A, et al. CARMELA Study Investigators. High prevalence of diabetes and impaired fasting glucose in urban Latin America: the CARMELA Study. Diabet Med. 2009; 26 (9): 864-71

2. Aschner P, King H, De Torrado M, Rodríguez BM. Glucose intolerance in Colombia: a population based survey in an urban community. Diabet Care. 1993; 16 (1): $90-3$

3. Selvin E, Marinopoulos S, Berkenblit G, et al. Meta-analysis: glycosylated hemoglobin and cardiovascular disease in diabetes mellitus. Ann Intern Med. 2004; 141 (6): 421-31.

4. Fowler MJ. Microvascular and macrovascular complications of Diabetes. Clin Diabet. 2008; 26 (2): 77-82.

5. Jönsson B. The Economic impact of diabetes. Diabet care. 1998; 21(Suppl 3): C7-10.

6. Haynes RB, McDonald H, Garg AX, Montague P. Interventions for helping patients to follow prescriptions for medications. Cochrane Databa Syst Rev. 2002; (2): CD000011.

7. Organización Mundial de la Salud(OMS). La OMS alerta sobre riesgos de incumplimiento del tratamiento médico [serie en Internet]. Disponible en: www. infomed.sld.cu Consultado Octubre 2009.

8. Osuna M, Rivera MC, Bocanegra C, Lancheros A, Tovar H, Hernández JI, et al. Caracterización de la diabetes mellitus tipo 2 y el control metabólico en el paciente hospitalizado. Acta Méd Colomb. 2014; 39 (4): 344-51.

9. Skrtic S, Cabrera C, Olsson M, Schnecke V, Lind M. Contemporary risk estimates of three $\mathrm{HbA} 1 \mathrm{c}$ variables in relation to heart failure following diagnosis of type 2 diabetes. Heart. 2017; 103 (5): 353-8.

10. Tomova G, Nimbal V, Horwich TB. Relation between hemoglobin A1c and outcomes in heart failure with or without diabetes mellitus. Am J Cardiol. 2012; 109 (12): 1767-73

11. Pérez M, Gómez-Arbelaez D, Melgarejo E, Bravo MA, Martínez A, González LA, et al. Hemoglobina glicosilada y su relación con la fracción de eyección del ventrículo izquierdo en pacientes diabéticos tipo 2 y un primer infarto agudo del miocardio. Rev Fac Med. 2014; 22 (2): 12-19.

12. Margolis KL, O'Connor PJ, Morgan TM, Buse JB, Cohen RM, Cushman WC, et al. Outcomes of Combined Cardiovascular Risk Factor Management Strategies in Type 2 Diabetes: The ACCORD Randomized Trial. Diabet Care. 2014; 37 (6): 1721-8.

13. Zoungas S, Chalmers J, Neal B, Billot L, Li Q, Hirakawa Y, et al. Follow-up of Blood-Pressure Lowering and Glucose Control in Type 2 Diabetes. N Engl J Med. 2014; 371 (15): 1392-406.

14. Bower JK, Appel LJ, Matsushita K, Young JH, Alonso A, Brancati FL, et al. Glycated hemoglobin and risk of hypertension in the atherosclerosis risk in communities study. Diabet Care. 2012; 35 (5): 1031-7.

15. Van Bruggen R, Gorter K, Stolk R, Zuithoff P, Klungel O, Rutten G. Refill adherence and polypharmacy among patients with type 2 diabetes in general practice. Pharmacoepidem drug safe. 2009; 18 (11): 983-91.

16. Figueroa CL, Gamarra G. Factores asociados con no control metabólico en diabéticos pertenecientes a un programa de riesgo cardiovascular. Acta méd Colomb. 2013; 38 (4): 213-21.

17. Odegard P, Capoccia K. Medication Taking and Diabetes: A Systematic Review of the Literature. Diabet Educ. 2007; 33 (6): 1014-29.

18. Hayward RA, Reaven PD, Wiitala WL, Bahn GD, Reda DJ, Ge L, et al. Follow-up of Glycemic Control and Cardiovascular Outcomes in Type 2 Diabetes. N Engl J Med. 2015; 372 (23): 2197-206.

19. The ADVANCE Collaborative Group, Patel A, MacMahon S, Chalmers J, Neal B, Billot L, et al. Intensive Blood Glucose Control and Vascular Outcomes in Patients with Type 2 Diabetes. N Engl J Med. 2008; 358 (24): 2560-72.

20. American Diabetes Association. Diagnosis and Classification of Diabetes Mellitus. Diabet Care. 2010; 33 (Suppl 1): S62-S69.

21. Masrur S, Cox M, Bhatt D, Smith E, Ellrodt G, Fonarow G. Association of Acute and Chronic Hyperglycemia With Acute Ischemic Stroke Outcomes PostThrombolysis: Findings From Get With The Guidelines-Stroke. J Am Heart Assoc. 2015; 4 (10): e002193.

22. Blasco ML, Sanjuan R, Palacios L, Huerta R, Carratala A, Nuñez J, et al.Prognostic value of admission glycated haemoglobin in unknown diabetic patients with acute myocardial infarction. Eur Heart $J$ Acute Cardiovasc Care. 2014; 3 (4): 347-53.

23. Figueiredo VN, Godoi FC, Martins NS, Quinaglia e Silva JC, Nadruz W, Coelho OR, et al. Diabetes mellitus unawareness is a strong determinant of mortality in patients manifesting myocardial infarction. Curr Med Res Opin. 2013; 29 (11): 1423-7.

24. Hoang QN, Pisani MA, Inzucchi S, Hu B, Honiden S. Prevalence of undiagnosed diabetes mellitus and the association of baseline glycemic control on mortality in the intensive care unit: A prospective observational study. J Crit Care. 2014; 29 (6): $1052-6$

25. Kompoti M, Michalia M, Salma V, Diogou E, Lakoumenta A, Clouva-Molyvdas PM. Glycated hemoglobin at admission in the ICU: clinical implications and prognostic relevance. J Crit Care. 2014; 30 (1): 150-5.

26. Ghaffari S, Niafar F, Separham A, Niafar M, Pourafkari L, Nader N. Association between HbA1c levels with severity of coronary artery disease and short-term outcomes of acute ST-elevation myocardial infarction in nondiabetic patients. Ther Adv Cardiovasc Dis. 2015; 9 (5): 305-13.

27. Tian L, Zhu J, Liu L, Liang Y, Li J, Yang Y. Hemoglobin A1c and short-term outcomes in patients with acute myocardial infarction undergoing primary angioplasty: an observational multicenter study. Coron Artery Dis. 2013; 24 (1): 16-22.

28. Fujino M, Ishihara M, Honda S, Kawakami S, Yamane T, Nagai T, et al. Impact of acute and chronic hyperglycemia on in-hospital outcomes of patients with acute myocardial infarction. Am J Cardiol. 2014; 114 (12): 1789-93. 